\title{
Clinical Burden of Modified Glasgow Prognostic Scale in Colorectal Cancer
}

\author{
YOSHINAGA OKUGAWA ${ }^{1,2,3^{*}}$, YUMIKO SHIRAI $^{4 *}$, YUJI TOIYAMA ${ }^{1}$, SUSUMU SAIGUSA $^{3}$, \\ ASAHI HISHIDA ${ }^{5}$, TAKESHI YOKOE ${ }^{3}$, KOJI TANAKA ${ }^{3}$, MOTOYOSHI TANAKA ${ }^{2}$, HIROMI YASUDA ${ }^{1}$, \\ HIROYUKI FUJIKAWA ${ }^{1}$, JUNICHIRO HIRO ${ }^{1}$, MINAKO KOBAYASHI ${ }^{1}$, TOSHIMITSU ARAKI ${ }^{1}$, \\ YASUHIRO INOUE ${ }^{1}$, DONALD C. MCMILLAN ${ }^{6}$, MASATO KUSUNOKI ${ }^{1}$ and CHIKAO MIKI ${ }^{3}$ \\ ${ }^{I}$ Department of Gastrointestinal and Pediatric Surgery, Division of Reparative Medicine, \\ Institute of Life Sciences, Mie University Graduate School of Medicine, Mie, Japan; \\ ${ }^{2}$ Department of Medical oncology, Iga General City Hospital, Mie, Japan; \\ ${ }^{3}$ Department of Surgery, Iga General City Hospital, Mie, Japan; \\ ${ }^{4}$ Department of Nutrition, Iga General City Hospital, Mie, Japan; \\ ${ }^{5}$ Department of Preventive Medicine, Nagoya University Graduate School of Medicine, Nagoya, Japan; \\ ${ }^{6}$ Academic Unit of Surgery, School of Medicine, University of Glasgow, Glasgow Royal Infirmary, Glasgow, U.K.
}

\begin{abstract}
Background/Aim: This study aimed to clarify the potential of modified Glasgow Prognostic Score ( $m G P S)$ as a prognostic biomarker and reveal the significance of fish oil (FO)-enriched nutrition in colorectal cancer (CRC). Patients and Methods: A total of 738 CRC patients from three different patient cohorts, including 670 patients in the biomarker study and 68 patients in the nutrition-intervention study, were analyzed. Results: High preoperative $m G P S$ was significantly correlated with wellrecognized disease progression factors and advanced UICC stage classification. In addition, high mGPS was an independent prognostic factor in both cohorts, especially in stage III and IV patients. These statuses were maintained in postoperative course and correlated with sarcopenia. Furthermore, FO-enriched nutrition suppressed systemic inflammatory reaction and improved skeletal muscle mass and prognosis, especially in CRC patients with $m G P S 1$ or 2. Conclusion: Assessment of mGPS could identify patients
\end{abstract}

\footnotetext{
*These Authors contributed equally to this study.

Correspondence to: Yoshinaga Okugawa, Department of Gastrointestinal and Pediatric Surgery, Division of Reparative Medicine, Institute of Life Sciences, Mie University Graduate School of Medicine, 2-174 Edobashi, Tsu, Mie 514-8507, Japan. Tel: +81 592315294, Fax: +81592326968, e-mail: yoshinaga.okugawa@ gmail.com; or Chikao Miki, Department of Surgery, Iga General City Hospital, 831, Shizuku-cho, Iga, Mie 518-0823, Japan. Tel: +81 595241111, e-mail: harborne3030@ gmail.com
}

Key Words: Colorectal cancer, modified Glasgow prognostic scale, prognosis, fish oil, eicosapentaenoic acid, nutrition. with high-risk CRC, who might be candidates for $\mathrm{FO}$ enriched nutrition.

Colorectal cancer (CRC) remains a leading cause of cancer mortality and the second leading cause of cancer-related deaths in the Western population (1). Despite recent advances in screening protocols, more than half of CRC cases have already reached an advanced stage where tumor cell spreading has occurred at the time of initial diagnosis $(2,3)$. Furthermore, although treatment options have significantly progressed, including the development of novel chemotherapeutic drugs and technical advances in the treatment of invasive metastatic lesions, approximately 50\% of CRC patients will die because of the development of systemic metastasis. Therefore, there is an urgent need for prognostic and predictive biomarkers in CRC patients and to identify appropriate patient groups for the most effective treatment options to improve disease outcomes.

In the last decade, accumulating evidence has revealed that cancer-associated inflammation is a key determinant of disease progression in various types of cancer $(4,5)$. Based on this evidence, it is currently well recognized that the hosttumor interaction in the form of the systemic inflammatory response (SIR) is intimately associated with outcomes in patients with cancer (6-8). The Glasgow Prognostic Score (GPS) is a previously defined inflammation-based scale based on serum levels of two acute phase proteins, $\mathrm{C}$-reactive protein (CRP) and albumin (Alb). Several studies successfully demonstrated the feasibility of using GPS as a prognostic biomarker for both operable and inoperable malignant disease (9). On further investigation, we defined the appropriate cut-off value of serum CRP levels as a 
prognostic biomarker for CRC patients in the Japanese population as $0.5 \mathrm{mg} / \mathrm{dl}$, which is termed the modified GPS (mGPS) $(10,11)$.

Previous work from our group has shown that several cytokines and serum markers reflecting systemic inflammatory response, including interleukin (IL)-1b, IL-1ra, IL-6, IL-10, $\mathrm{CRP}$, and Alb, are differentially expressed in serum from patients with advanced CRC and can be used as predictive biomarkers for postoperative nutritional status, morbidity, and mortality in CRC patients (12-16). Furthermore, we recently discovered the potential advantages of fish oil (FO)-enriched nutrition therapy in the chemotherapeutic treatment course of advanced gastrointestinal cancer (17). In the current study, we focused on the potential of our mGPS as a prognostic biomarker in patients with CRC, and systemically investigated the status of pre-operative mGPS in CRC patients using clinical data from two independent large patient cohorts to determine the clinical significance of mGPS in CRC. In addition, we evaluated the dysregulated pattern of serum CRP and albumin levels during the post-operative course in CRC patients to clarify the correlation between pre- and postoperative serum levels of these factors and tumor progression in CRC patients. Furthermore, we focused on the potential advantages of fish oil (FO)-enriched nutrition therapy as an anti-SIR nutrition intervention in the treatment course of patients with advanced CRC. We assessed the chronological alterations of biochemical and physiological status during chemotherapeutic treatment with or without FOenriched nutrition intervention in patients with advanced CRC to clarify the clinical benefit of FO-enriched nutrition for advanced CRC.

\section{Patients and Methods}

A total of 738 patients with CRC from three different patient cohorts, including 670 patients in the biomarker study phase and 68 in the nutrition intervention study phase, were analyzed (Table I). The retrospective study design included an initial biomarker study phase with two separate cohorts, followed by a nutrition intervention study phase with the third cohort (17). The training cohort of the biomarker study phase was composed of 125 patients with primary CRC who underwent surgical treatment at the Department of Surgery, Iga Ueno General Hospital (Iga, Mie, Japan) between January 2008 and December 2012. The validation phase of the biomarker study phase involved 545 CRC patients who underwent surgical treatment at the Department of Gastrointestinal Surgery, Mie University Hospital (Iga, Mie, Japan) between January 2000 and December 2011. The nutrition intervention study cohort contained 68 patients who received therapeutic chemotherapy for advanced or recurrent CRC at the Department of Medical Oncology in Iga Municipal Ueno General Citizen's Hospital (Iga, Mie, Japan) between April 2011 and April 2014. In the biomarker study cohort, the Tumor Node Metastasis (TNM) staging system from the American Joint Committee on Cancer was used for pathological tumor staging of colorectal cancer (18). Resection of the primary tumor was performed in all patients, and all patients were followed up for tumor recurrence at regular intervals for up to 5 years. No perioperative mortalities were observed among these patients. No patient had received chemotherapy or radiation therapy prior to surgery. During each annual hospital visit, all patients underwent a chest X-ray, colonoscopy, and abdominal computed tomography. Patients who were treated with radiotherapy or chemotherapy before surgery were excluded from this study. All CRC patients with stage III/IV disease received 5-fluorouracil-based chemotherapy, whereas no adjuvant chemotherapy was given to patients in stage I or II. The diagnosis of CRC was confirmed for all enrolled patients based on clinicopathological findings. Patients were observed at 3-month intervals for 24 months after completion of surgery, every 6 months for the following 3 years, and yearly thereafter. A history was taken and a physical examination was performed at each visit, and chest $\mathrm{X}$-ray, colonoscopy, and CT were performed annually.

In the nutrition intervention study, 68 patients with CRC who visited the outpatient clinic for cancer chemotherapy at Iga General Hospital were enrolled. Eligibility criteria for recruitment were as follows: clinical diagnosis of colorectal cancer with stage III or IV (by radiological/histological/cytological confirmation); patient between the ages of 18 and 80 years; $5 \%$ or greater loss of pre-illness body weight; life expectancy of 3 months or longer; Karnofsky performance status of 70 or higher (19). All patients underwent adjuvant or palliative chemotherapy (17 patients had adjuvant chemotherapy and 51 had palliative chemotherapy). Body composition measurements including total body weight, skeletal muscle mass, body fat quantity, body fat percentage, lean body mass, and extracellular/total body water were measured every 3 months at each visit to this clinic. Patients were randomly assigned to a treatment course with or without FO-enriched nutrition by the healthcare staff. Patients with FO-enriched nutrition received one or two packs of FO-enriched oral nutritional supplements per day according to their tolerance of FO-enriched nutrition (Prosure ${ }^{\circledR}$, Abbott Nutrition, Tokyo, Japan) and took the product for 6 months. One pack contains $16 \mathrm{~g}$ of protein, $1.1 \mathrm{~g}$ of EPA, $0.5 \mathrm{~g}$ of DHA, and a total of $355 \mathrm{kcal}$. Patients who did not receive FOenriched nutrition were not given any additional nutritional treatment or placebo, as previously described (17). Patient characteristics are summarized in Table I. Written informed consent was obtained from all patients, and the study was approved by the institutional review boards of all participating institutions.

Imaging analysis. Preoperative and postoperative (6 months after surgery) CT imaging was performed with a multidetector CT scanner (Aquilion 64, Toshiba Medical Systems, Tochigi, Japan). The technical parameters used for CT were as follows: $135 \mathrm{kV}$ tube voltage, $0.5 \mathrm{~mm} \times 64$ row detector configuration, tube current modulation, $0.5 \mathrm{~s} /$ rotation gantry rotation, and $4 \mathrm{~mm}$ reconstruction thickness. Using plain CT images at the level of the caudal end of the third lumbar vertebra, the cross-sectional areas of the bilateral psoas muscles were measured by manual tracing.

Measurements. In the nutrition intervention study routine laboratory measurements, including serum C-reactive protein (CRP) and albumin (Alb), were carried out on the same day. At that time, no patients showed clinical evidence of infection. Serum carcinoembryonic antigen (CEA) and CA19-9 were also measured routinely at the same time by enzyme immunoassay on a Tosoh AIA 21 (Diamond Diagnostics, Holliston, MA, USA). The analysis of body composition included total body weight, height, body mass index (BMI), lean body mass, and fat mass. Skeletal muscle mass, body fat quantity, body fat percentage, lean body mass, and extracellular/total body water were measured using bioelectrical 
Table I. Characteristics of all patients from each CRC cohorts.

\begin{tabular}{|c|c|c|c|c|}
\hline & \multicolumn{2}{|c|}{ Biomarker phase } & \multicolumn{2}{|c|}{ Nutrition intervention phase } \\
\hline & $\begin{array}{l}\text { Iga Ueno Cohort } \\
\qquad(\mathrm{n}=125)\end{array}$ & $\begin{array}{l}\text { Mie University Cohort } \\
\qquad(\mathrm{n}=545)\end{array}$ & $\begin{array}{l}\text { With nutrition intervention } \\
\qquad(\mathrm{n}=15)\end{array}$ & $\begin{array}{l}\text { Without nutrition intervention } \\
\qquad(\mathrm{n}=53)\end{array}$ \\
\hline \multicolumn{5}{|l|}{ Gender } \\
\hline Male & 71 & 321 & 11 & 35 \\
\hline Female & 54 & 224 & 4 & 18 \\
\hline \multicolumn{5}{|l|}{ Age (y) } \\
\hline$(\mathrm{Mean} \pm \mathrm{SD})$ & $72.7 \pm 11.9$ & $66.9 \pm 11.3$ & $72.1 \pm 7.9$ & $68.9 \pm 11.1$ \\
\hline \multicolumn{5}{|l|}{ Tumor Location } \\
\hline Colon & 86 & 321 & 9 & 30 \\
\hline Rectum & 39 & 224 & 6 & 23 \\
\hline \multicolumn{5}{|l|}{ Histologycal type } \\
\hline Differentiated & 115 & 467 & & \\
\hline Undifferentiated & 10 & 78 & & \\
\hline \multicolumn{5}{|l|}{ TNM classification } \\
\hline Stage I & 16 & 123 & 0 & 0 \\
\hline Stage II & 34 & 162 & 0 & 0 \\
\hline Stage III & 46 & 135 & 4 & 13 \\
\hline Stage IV & 28 & 125 & 11 & 40 \\
\hline
\end{tabular}

impedance (InBody ${ }^{\circledR}$, Cerritos, CA, USA) every 3 months as previously described (17).

The modified Glasgow Prognostic Score (mGPS) was determined as previously described $(10,20)$. Briefly, patients with elevated Creactive protein $(>0.5 \mathrm{mg} / \mathrm{dl})$ were assigned a score of 1 or 2 depending on the absence or presence of hypoalbuminemia $(<3.5$ $\mathrm{g} / \mathrm{dl}$ ) respectively. Patients with normal CRP and any albumin level were given a score of 0 .

Statistical methods. Statistical analysis was performed using Medcalc version 16.8.4 (Broekstraat 52, 9030; Mariakerke, Belgium). Results are expressed as means+standard deviation (SD). Differences between groups were estimated by the Chi-Square test, one-way ANOVA analysis, and Friedman test as appropriate. F-tests were conducted to assess the equality of variance for comparable groups. For time-to-event analyses, survival estimates were calculated using Kaplan-Meier analysis, and groups were compared with the log-rank test. Overall survival (OS) was measured from the date the patient underwent surgery until the date of death from any cause (i.e., cancer-unrelated deaths were not censored), or the last known follow-up for patients that were still alive. Disease-free survival (DFS) was measured from the date the patient underwent curative surgery to the date of disease recurrence, death from any cause (i.e., cancer unrelated deaths were not censored), or until last contact with the patient. Cox's proportional hazards models were used to estimate hazard ratios (HR) for death. Assumption of proportionality was confirmed for the Cox proportional hazards analyses by generating Kaplan-Meier survival curves (e.g., with vs. without FO-enriched nutrition groups) and ensuring that the two curves did not intersect each other. Forced-entry regression was used to include these variables in all multivariable equations to analyze whether each of the predictors affecting the outcome after adjusting for known confounders. Clinical variables that were considered for univariate and multivariate analyses, in addition to target mGPS status, were previously identified confounding factors that affected the prognosis of patients with colorectal cancer: sex, age at diagnosis, tumor location (colon or rectum), pathological differentiation (well-moderate or poor), T-stage (T1/2 or T3/4), venous invasion (present or absent), lymphatic vessel invasion (present or absent), lymph node metastasis (present or absent), liver metastasis (presence or absence), and distant metastasis (presence or absence). Tests for interaction were performed by the likelihood ratio test of cross-product terms. All $p$-values were 2 -sided, and values less than 0.05 were considered statistically significant.

\section{Results}

Preoperative mGPS score was significantly increased concurrent with disease progression in CRC patients from Iga Ueno cohort. First, we analyzed the correlation between clinicopathological factors and preoperative mGPS status in CRC patients from the training cohort (Table II). In addition to older age and tumor location, increased preoperative mGPS score was significantly associated with all wellestablished disease progression clinicopathological factors, including advanced T stage $(p=0.008)$, presence of venous invasion $(p=0.02)$, lymphatic vessel invasion $(p=0.01)$, lymph node metastasis $(p=0.03)$, distant metastasis $(p=0.005)$, and advanced TNM stage classification $(p=0.0009)$ in CRC patients from the training cohort.

High score of preoperative mGPS was associated with poor outcome in CRC patients from Iga Ueno cohort. Next, for timeto-event analysis to evaluate the potential of preoperative mGPS score as a prognostic biomarker we generated Kaplan-Meier survival curves subdivided by mGPS score. Interestingly, patients with increased mGPS score had a significantly poorer 
Table II. Clinicopathological variables and preoperative mGPS status in CRC patients from two independent cohorts.

\begin{tabular}{|c|c|c|c|c|c|c|c|c|c|c|}
\hline \multirow[t]{3}{*}{ Variable } & \multicolumn{4}{|c|}{ Iga Ueno Cohort } & \multirow[b]{3}{*}{$p$-Value } & \multicolumn{4}{|c|}{ Mie University Cohort } & \multirow[b]{3}{*}{$p$-Value } \\
\hline & \multicolumn{4}{|c|}{ Preoperative mGPS } & & \multicolumn{4}{|c|}{ Preoperative mGPS } & \\
\hline & $\mathrm{n}$ & $\begin{array}{c}0 \\
(\mathrm{n}=68)\end{array}$ & $\begin{array}{c}1 \\
(n=26)\end{array}$ & $\begin{array}{c}2 \\
(n=31)\end{array}$ & & $\mathrm{n}$ & $\begin{array}{c}0 \\
(\mathrm{n}=373)\end{array}$ & $\begin{array}{c}1 \\
(\mathrm{n}=97)\end{array}$ & $\begin{array}{c}2 \\
(\mathrm{n}=75)\end{array}$ & \\
\hline \multicolumn{11}{|l|}{ Gender } \\
\hline Male & 71 & 38 & 14 & 19 & 0.66 & 321 & 218 & 62 & 41 & $0.84^{\#}$ \\
\hline Female & 54 & 30 & 12 & 12 & & 224 & 155 & 35 & 34 & \\
\hline \multicolumn{11}{|l|}{ Age $(y)$} \\
\hline Mean \pm SD & 125 & $70.5 \pm 11.7$ & $73.2 \pm 12.6$ & $77.0 \pm 10.9$ & $0.014 *$ & 545 & $66.9 \pm 11.5$ & $65.6 \pm 10.1$ & $68.3 \pm 11.7$ & $0.31^{\# \#}$ \\
\hline \multicolumn{11}{|l|}{ Location } \\
\hline Colon & 86 & 39 & 21 & 26 & $0.004 *$ & 321 & 208 & 64 & 49 & $0.047^{*}$ \\
\hline Rectum & 39 & 29 & 5 & 5 & & 224 & 165 & 33 & 26 & \\
\hline \multicolumn{11}{|l|}{ Histological type } \\
\hline Differentiated & 115 & 64 & 23 & 28 & 0.44 & 467 & 326 & 81 & 60 & $0.07^{\#}$ \\
\hline Undifferentiated & 10 & 4 & 3 & 3 & & 78 & 47 & 16 & 15 & \\
\hline \multicolumn{11}{|c|}{ Pathological $\mathrm{T}$ category } \\
\hline $\mathrm{T} 1 / \mathrm{T} 2$ & 20 & 17 & 1 & 2 & $0.008^{*}$ & 163 & 144 & 10 & 9 & $<0.0001^{* A}$ \\
\hline $\mathrm{T} 3 / \mathrm{T} 4$ & 105 & 51 & 25 & 29 & & 382 & 229 & 87 & 66 & \\
\hline \multicolumn{11}{|l|}{ Venous invasion } \\
\hline+ & 57 & 22 & 19 & 16 & $0.02 *$ & 302 & 188 & 65 & 49 & $0.002^{* \#}$ \\
\hline- & 68 & 46 & 7 & 15 & & 243 & 185 & 32 & 26 & \\
\hline \multicolumn{11}{|l|}{ Lymphatic invasion } \\
\hline+ & 98 & 46 & 25 & 27 & $0.01 *$ & 428 & 274 & 85 & 69 & $<0.0001^{* A}$ \\
\hline- & 27 & 22 & 1 & 4 & & 117 & 99 & 12 & 6 & \\
\hline \multicolumn{11}{|c|}{ Lymph node metastasis } \\
\hline+ & 68 & 31 & 16 & 21 & $0.03 *$ & 227 & 138 & 51 & 38 & $0.004^{* \#}$ \\
\hline- & 57 & 37 & 10 & 10 & & 318 & 235 & 46 & 37 & \\
\hline \multicolumn{11}{|l|}{ Distant metastasis } \\
\hline+ & 16 & 4 & 4 & 8 & $0.005^{*}$ & 125 & 59 & 38 & 28 & $<0.0001 * \hbar$ \\
\hline- & 109 & 64 & 22 & 23 & & 420 & 314 & 59 & 47 & \\
\hline \multicolumn{11}{|c|}{ UICC stage classification } \\
\hline Stage I & 16 & 13 & 1 & 2 & $0.0009^{*}$ & 123 & 109 & 8 & 6 & $<0.0001^{* \#}$ \\
\hline Stage II & 34 & 22 & 7 & 5 & & 162 & 105 & 30 & 27 & \\
\hline Stage III & 46 & 26 & 11 & 9 & & 135 & 100 & 21 & 14 & \\
\hline Stage IV & 28 & 6 & 7 & 15 & & 125 & 59 & 38 & 28 & \\
\hline
\end{tabular}

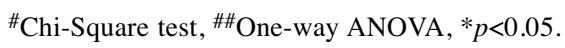

prognosis than those with low mGPS score in terms of overall survival (OS; log-rank test, $p<0.0001$, Figure 1a). Multivariate Cox's regression analysis to determine the potential of preoperative mGPS score as a predictive biomarker of prognosis in CRC patients showed that elevated mGPS score and presence of distant metastasis were independent prognostic factors for OS $\quad(\mathrm{HR}=1.68,95 \% \mathrm{CI}=1.21-2.32, \quad p=0.002, \quad \mathrm{HR}=2.51$, $95 \% \mathrm{CI}=1.12-5.6, p=0.025$, respectively) in CRC patients from the Iga Ueno cohort (Table III).

Clinical significance of preoperative mGPS status in CRC patients from Mie University cohort. To confirm the clinical significance of preoperative mGPS status in CRC patients, we validated our findings in an additional independent large CRC cohort. Consistent with the clinical findings for the training cohort from Iga Ueno general hospital, high mGPS score was correlated with tumor location of colon $(p=0.047)$, advanced T stage $(p<0.0001)$, presence of venous invasion $(p=0.002)$, lymphatic vessel invasion $(p<0.0001)$, lymph node metastasis $(p=0.004)$, distant metastasis $(p<0.0001)$, and advanced UICC stage classification $(p<0.0001)$ in CRC patients from the Mie University cohort. Furthermore, regarding survival outcomes in the Mie University cohort, mGPS score was significantly associated with prognosis with regard to OS and DFS in a mGPS score-dependent manner (log rank test $p<0.0001, p<0.003$, respectively; Figure 1b). Furthermore, multivariate Cox's regression analysis revealed that high mGPS score was an independent predictor for poor prognosis of OS in the Mie University cohort $(\mathrm{HR}=1.95$, $95 \% \mathrm{CI}=1.55-2.45, p<0.0001$, Table IV). 
Preoperative mGPS score could identify high-risk stage III/IV CRC patients. To determine the prognostic impact of preoperative mGPS score as a predictive biomarker of prognosis in stage III and IV CRC patients who require postoperative chemotherapy, we evaluated survival outcomes in stage III and IV patients subdivided according to preoperative mGPS score (shown in Figure 1c, d). Elevated mGPS score was associated with poor OS in patients belonging to the Iga Ueno cohort and the Mie University cohort $(p<0.0001$ and $p<0.0001$, respectively, log-rank test). Furthermore, multivariate analysis revealed that high mGPS score was an independent predictor for poor prognosis of OS in both Iga Ueno and Mie University cohorts $(\mathrm{HR}=1.55,95 \% \mathrm{CI}=1.09-2.21, p=0.015 ; \quad \mathrm{HR}=2$, $95 \% \mathrm{CI}=1.51-2.65, p<0.0001$, respectively, Tables $\mathrm{V}$ and VI). Interestingly, mGPS score was not significantly correlated with poor survival in CRC patients with stage II disease in both cohorts ( $p=0.25,0.19$, respectively, log rank test, Figure 1e, f). Collectively, our data revealed that preoperative mGPS score could identify high-risk patients, especially among those with stage III and IV CRC.

Preoperative CRP and albumin status was positively correlated with postoperative levels of each factor and tumor progression in CRC patients. To clarify the dysregulation of $\mathrm{CRP}$ and Alb levels during the long-term postoperative course in patients with elevated CRP levels or hypoalbuminemia, we evaluated serum CRP and Alb levels 3 and 6 months after surgery in CRC patients from the Iga Ueno cohort. Interestingly, serum CRP concentration 3 or 6 months after surgery was significantly elevated in CRC patients with preoperative high CRP levels $(p=0.0016, \quad p=0.0005$, respectively, Table VII). Similarly, serum Alb concentration 3 or 6 months after surgery was significantly decreased in CRC patients with preoperative hypoalbuminemia $(p=0.0004$, $p=0.0019$, respectively, Table VII). Furthermore, postoperative serum levels of CRP and Alb were significantly correlated with serum CEA levels 3 and 6 months after surgery (Alb: $p<0.0001, \mathrm{r}=-0.54, p<0.0001, \mathrm{r}=-0.58$, respectively; CRP: $p<0.0001, \mathrm{r}=0.73, p=0.0002, \mathrm{r}=0.5$, respectively).

Preoperative high-CRP or low-albumin status correlated with pre-and postoperative sarcopenia in CRC patients. Based on findings of biochemical dysregulation in CRC patients, we next evaluated pre- and postoperative skeletal muscle mass 6 months after surgery using CT imaging analysis to clarify the correlation between preoperative CRP and albumin status and perioperative quantity of skeletal muscle mass in CRC patients from the Iga Ueno cohort (Table VIII). Preoperative skeletal muscle mass was significantly decreased in patients with high preoperative CRP status $(p=0.024)$. In addition, postoperative skeletal muscle mass was significantly decreased in patients with preoperative hypoalbuminemia $(p=0.033)$, and tended to be decreased in the preoperative high-CRP group $(p=0.058)$. Collectively, these findings revealed that a preoperative status of elevated CRP level and hypoalbuminemia was significantly correlated with a dysregulated pattern of each factor and tumor development during the postoperative course. Moreover, these factors were significantly correlated with perioperative cachexia in CRC patients.

FO-enriched nutrition inhibited the increase in serum CRP levels and led to improvement of skeletal muscle mass and lean body mass in colorectal cancer patients. N-3 (omega-3) polyunsaturated fatty acids (FAs) from fish oil (FO) have plausible immune-modulating effects with a low proinflammatory and immunosuppressive effect, in part due to the formation of 3-series prostanoids and 5-series leukotrienes $(21,22)$. In particular, eicosapentaenoic acid (EPA) is a naturally occurring N-3 fatty acid present in FO that has been shown to have anti-inflammatory properties and anti-cachexia activity via suppression of interleukin (IL)-6 production, downregulation of the acute phase response, and a reduction in serum concentration of CRP in various type of cancer (20, 23). Furthermore, recent evidence demonstrated that cancer cachexia, which is induced by the SIR via disease progression, decreases the tolerance for chemotherapeutic treatment and worsens the prognosis in CRC patients (24-26). However, the impact of FO-enriched nutrition as a combined modality therapy on clinical outcomes remains controversial. Based on the above evidence, we systemically investigated the chronological alteration of biochemical and physiological status during chemotherapeutic treatment with or without FOenriched nutrition in CRC patients to clarify the clinical significance and prognostic benefits of FO-enriched nutritional therapy in the treatment course of advanced CRC.

Body mass index (BMI) and extracellular water ratio (extracellular water (ECW)/total body water (TBW)) showed no significant differences between patients with or without FO-enriched nutrition during the course of the study (data not shown). Serum CRP levels gradually increased in a timedependent manner in patients without FO-enriched nutrition $(p=0.39)$, but gradually decreased in patients with FOenriched nutrition $(p=0.24)$. Furthermore, significant increases in skeletal muscle mass and lean body mass were observed in patients with FO-enriched nutrition $(p<0.0001$, $p<0.0001$, respectively, Figure 2), but both factors showed no significant improvement in patients without FO-enriched nutrition ( $p=0.14, p=0.06$, respectively).

FO-enriched nutrition significantly improved prognosis in colorectal cancer patients with mGPS 1 or 2 . Finally, we evaluated the prognostic impact of FO-enriched nutrition in CRC patients. Unfortunately, survival curve analysis in total cohorts showed no significant difference between patient groups with or without FO-enriched nutrition $(p=0.99$, Figure 
a

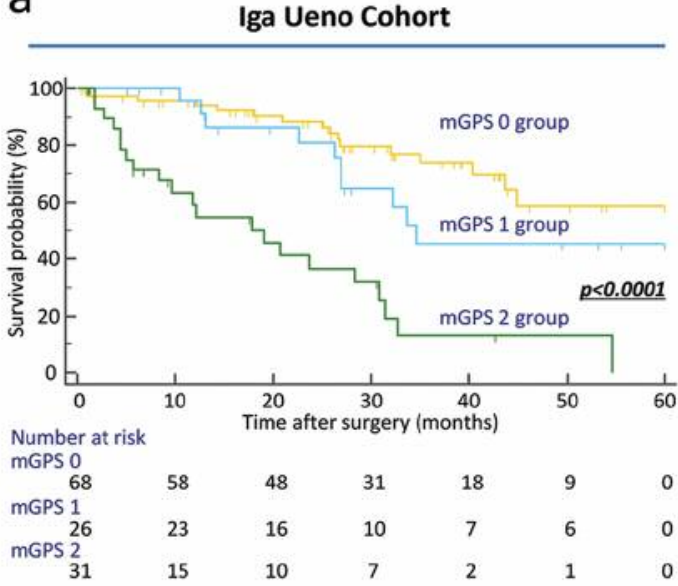

C

Iga Ueno Cohort

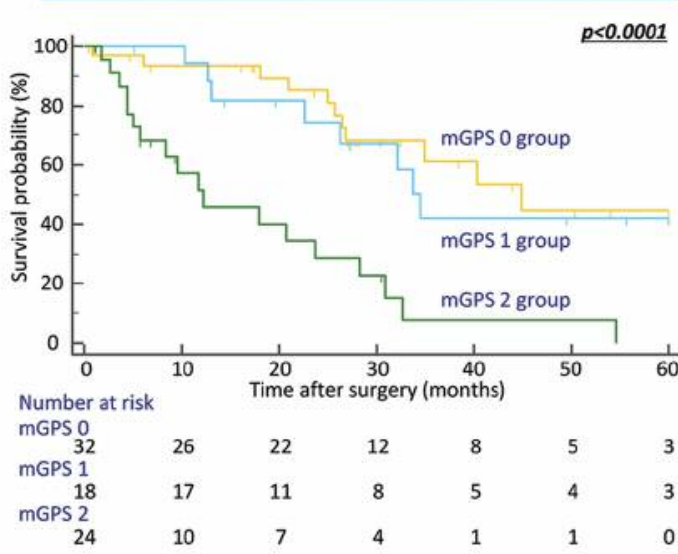

e

Iga Ueno Cohort

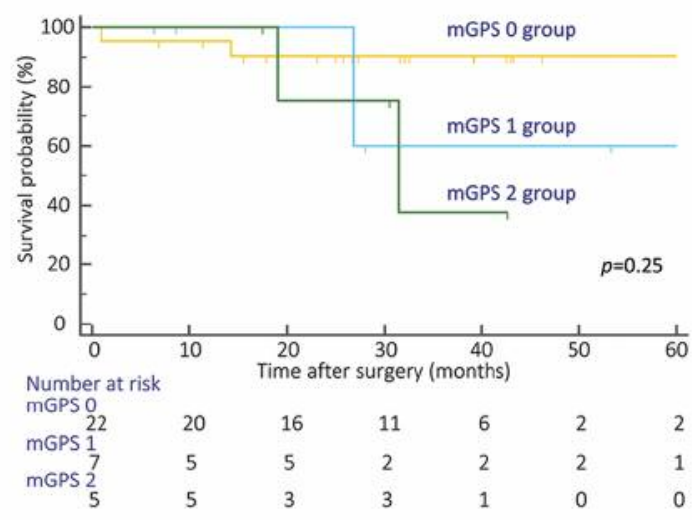

b

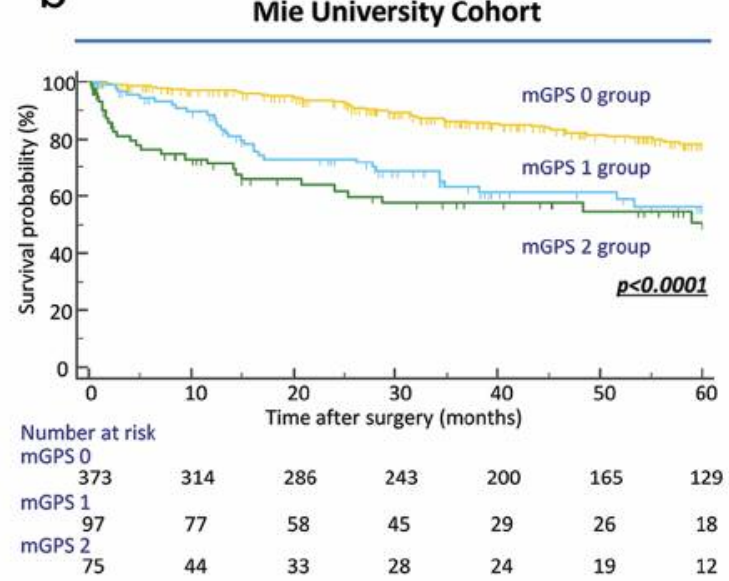

d Mie University Cohort

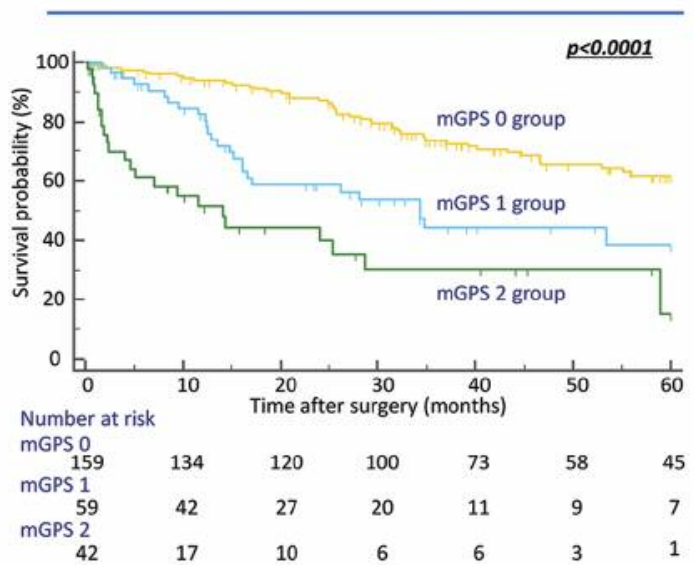

$f$

Mie University Cohort

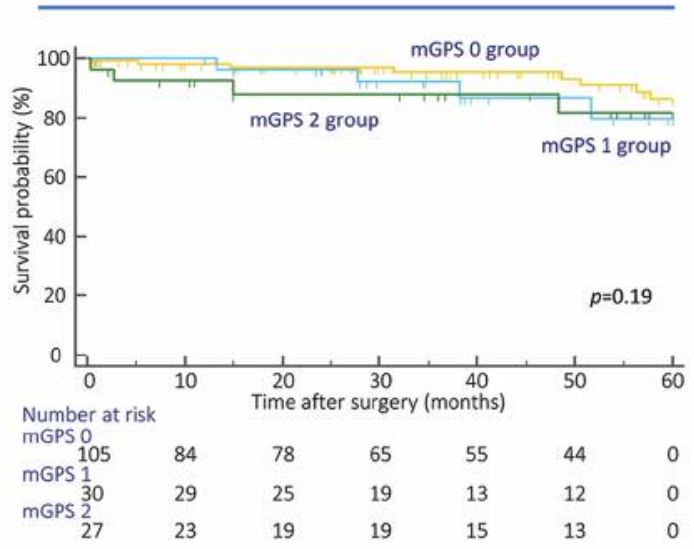

Figure 1. Prognostic impact of mGPS status for overall survival of CRC patients from the Iga Ueno cohort and Mie University Cohort. Kaplan-Meier survival curves for overall survival (OS) in CRC patients according to $M G P S$ score in the Iga Ueno cohort (a) and Mie University cohort (b). The OS rate in CRC patients with high preoperative mGPS score was significantly lower than that of patients with low mGPS score $(p<0.0001, p<0.0001$, respectively, log-rank test). All statistical tests were two-sided. Kaplan-Meier survival curves for overall survival (OS) in CRC patients with UICC stage III and IV disease according to mGPS score in Iga Ueno cohort (c) and Mie University cohort (d). The OS rate in CRC patients with high preoperative $m G P S$ score was significantly lower than that of patients with low $m G P S$ score $(p<0.0001, p<0.0001$, respectively, log-rank test). (e, $f)$ Survival curve analysis according to preoperative mGPS in CRC patients with UICC stage II disease. Kaplan-Meier survival curves for overall survival (OS) in CRC patients with UICC stage III and IV disease according to mGPS score in Iga Ueno cohort (e) and Mie University cohort (f). All statistical tests were two-sided. 

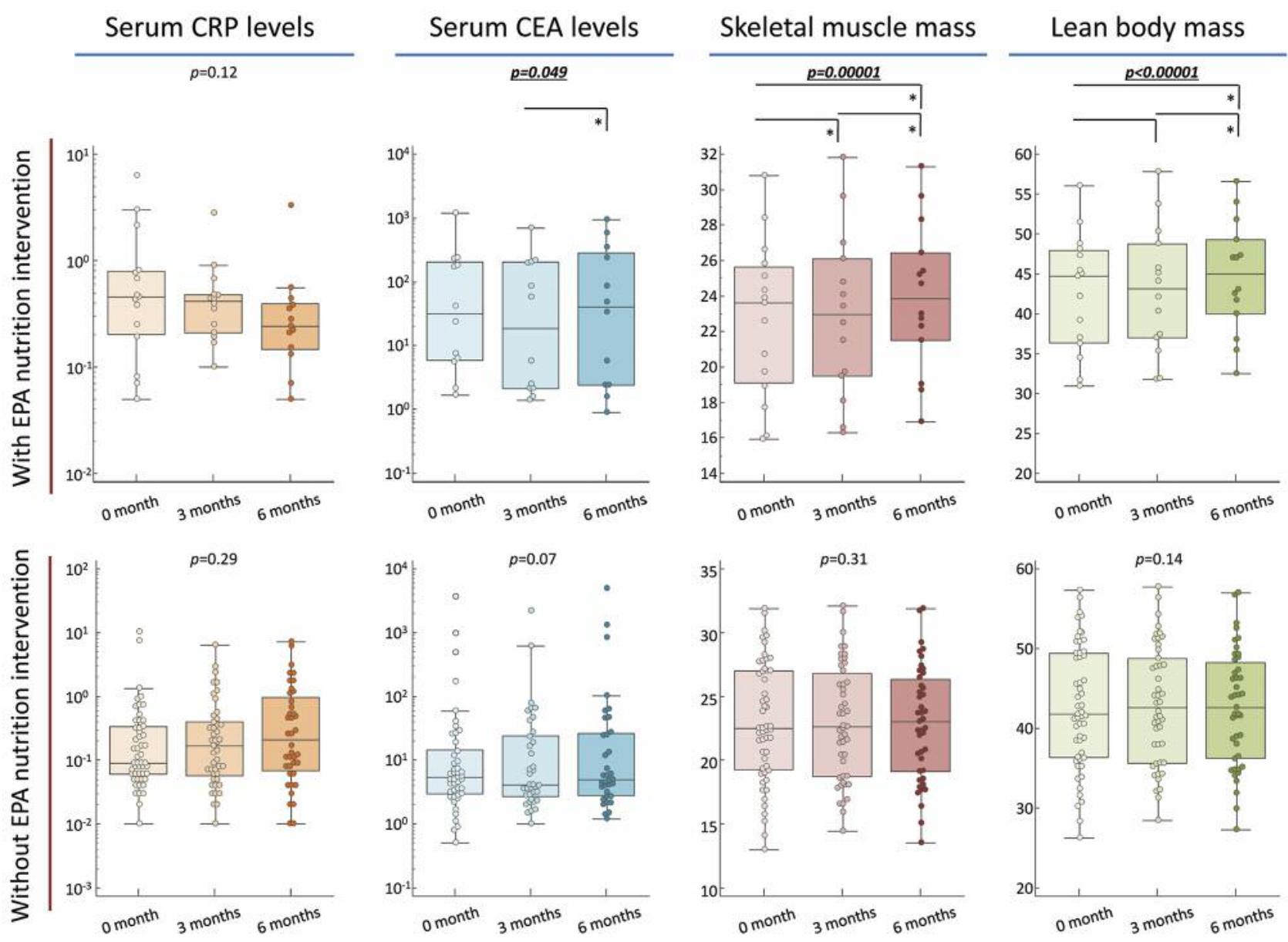

Figure 2. Chronological alterations in biochemical and physiological status during systemic chemotherapy in CRC patients. Upper line: CRC patients with FO-enriched nutrition, Lower line: CRC patients without FO-enriched nutrition. Box plots show biochemical (serum CRP and CEA levels) and physiological status (skeletal muscle mass and lean body mass) of CRC patients with FO-enriched nutrition during systemic chemotherapy. Although serum CEA levels significantly increased over 6 months ( $p=0.036$ ), serum CRP levels gradually decreased in the FO-enriched nutritional intervention group. Furthermore, there was a significant improvement in skeletal muscle mass and lean body mass in the FO-enriched nutritional intervention group $\left(p<0.0001, p<0.0001\right.$, respectively. Statistical analysis was performed using Friedman tests. All statistical tests were two-sided. ${ }^{*} p<0.05$.

3a). However, in subgroup analysis the groups with mGPS 1 or 2 showed significantly better prognosis when they received FO-enriched nutrition ( $p=0.035$, Figure $3 b)$. Collectively, our data suggest that FO-enriched nutrition might improve prognosis in CRC patients with SIR (mGPS of 1 or 2).

\section{Discussion}

A growing number of studies have demonstrated the potential application of mGPS as a prognostic biomarker in various types of cancer. However, almost all these data were from single-institute studies, and the feasibility of preoperative mGPS as a prognostic indicator and its clinical significance in advanced CRC using clinical data from multiple independent large cohorts has never been reported.
Furthermore, the clinical benefit of FO-enriched nutrition as an anti-SIR nutritional intervention remains controversial in CRC patients. From these standpoints, this investigation provided several important discoveries. First, a high preoperative mGPS score was significantly correlated with well-recognized disease progression factors, including advanced T-stage, presence of venous invasion, lymphatic vessel invasion, lymph node metastasis, distant metastasis, and advanced UICC stage classification in both cohorts of CRC patients. In addition, high mGPS status was one of the independent prognostic factors in both cohorts, and these data clearly re-confirmed preoperative mGPS as a feasible prognostic marker in CRC patients. Second, a high score of preoperative mGPS was an independent prognostic factor for survival, especially in CRC patients with stage III and IV 
a

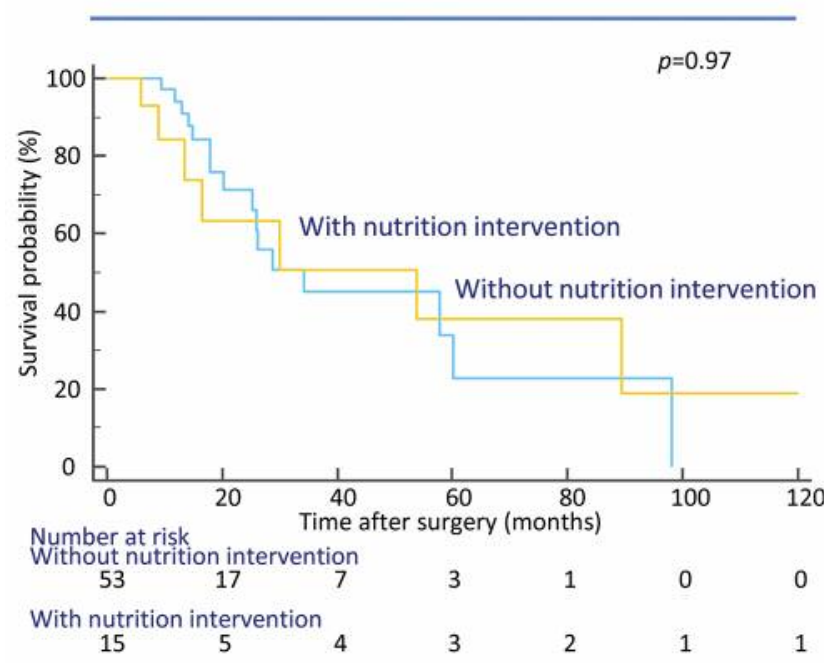

b CRC patients with $\mathrm{mGPS} 1$ and 2 patients

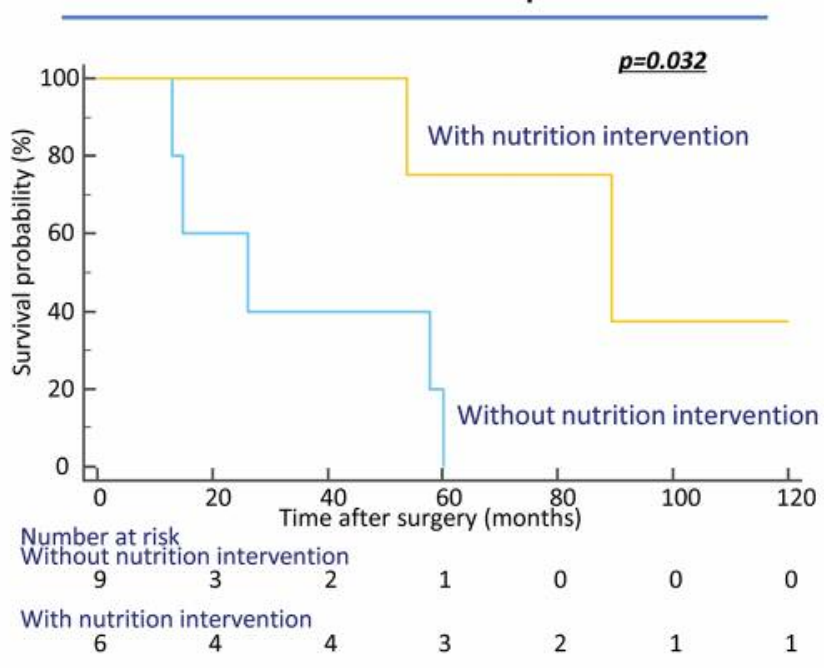

Figure 3. Prognostic impact of nutritional intervention with FO treatment in CRC patients. (a) Kaplan-Meier survival curves of overall survival in advanced CRC patients according to treatment with nutrition intervention in the total cohort $(n=68)$. The overall survival rate in CRC patients with FO-enriched nutritional intervention ( $n=15)$ was not statistically significantly different from that of patients without FO-enriched nutritional intervention ( $n=53)$ ( $p=0.99$; log-rank test). (b) Overall survival analyses of CRC patients with mGPS scores 1 and 2 according to treatment with EPA-enriched nutrition. CRC patients with mGPS 1 or 2 who received FO-enriched nutrition showed better prognosis than patients without FOenriched nutrition ( $p=0.035$; log-rank test). All statistical tests were two-sided.

patients, and this finding was successfully validated in an independent large CRC cohort. Third, serum CRP concentration 3 or 6 months after surgery was significantly elevated in CRC patients with preoperative high CRP levels compared with patients with low CRP levels, and serum Alb concentration showed a similar trend. Furthermore, these factors were significantly correlated with tumor progression and sarcopenia during the postoperative course in CRC patients. Fourth, although serum CEA levels significantly increased in a time-dependent manner during systemic chemotherapy, serum CRP levels gradually decreased in CRC patients with FO-enriched nutrition intervention. Furthermore, both skeletal muscle mass and lean body mass increased significantly even under systemic chemotherapy in the FO-enriched nutrition group, but showed no significant changes in CRC patients without FO-enriched nutrition. Finally, FO-enriched nutrition significantly improved prognosis for overall survival in CRC patients with SIR (mGPS 1 or 2).

C-reactive protein (CRP) is an acute-phase protein, and is produced in hepatocytes via activation of IL-6 and tumor necrosis factor- $\alpha$ (27). Previously, Macmillan and coworkers revealed that elevated levels of preoperative CRP are significantly correlated with an increased risk for early recurrence and poor outcome in CRC patients (28-31). In contrast, several studies demonstrated an intimate correlation between preoperative hypoalbuminemia and poor outcome in CRC patients (32-34). Heys and colleagues evaluated preoperative serum albumin levels using clinical data from 431 CRC patients, and showed that preoperative hypoalbuminemia was an independent prognostic indicator in patients with localized CRC (33). Based on these findings, Forrest and colleagues first developed a combined assessment of serum CRP and albumin levels, termed the GPS, as a prognostic indicator in patients with non-small cell lung cancer (35). Further studies successfully demonstrated the potential feasibility of GPS as a prognostic biomarker in various types of cancer, including CRC (9). In this study, we used our developed modified GPS, defined according to our previous studies (10, 11), rather than the original GPS and the cut-off value for abnormal serum CRP level was set at $0.5 \mathrm{mg} / \mathrm{dl}$. One of the major findings in this study was the prognostic impact of our mGPS in CRC patients; our assessment using two independent large cohorts clearly revealed that a high preoperative mGPS score was significantly correlated with all of the wellrecognized disease progression factors and poor prognosis in both cohorts. Especially, high preoperative mGPS was an independent prognostic factor in CRC patients with stage III and IV disease, but not stage II, and this finding was successfully validated in an independent large CRC cohort. These findings suggested that preoperative mGPS could identify high-risk patients, especially among those with advanced CRC. 
Table III. Multivariate analysis for predictors of overall survival in Iga Ueno cohort.

\begin{tabular}{|c|c|c|c|c|c|c|}
\hline \multirow[t]{2}{*}{ Variables } & \multicolumn{3}{|c|}{ Univariate } & \multicolumn{3}{|c|}{ Multivariate } \\
\hline & HR & $95 \% \mathrm{CI}$ & $p$-Value & HR & $95 \% \mathrm{CI}$ & $p$-Value \\
\hline Gender (Male) & 1.42 & $0.78-2.55$ & 0.25 & 1.08 & $0.56-2.11$ & 0.81 \\
\hline Age $\geq 74(\text { median})^{\#}$ & 1.08 & $0.61-1.91$ & 0.78 & 1.11 & $0.55-2.23$ & 0.78 \\
\hline Tumor location (Rectum) & 1.35 & $0.76-2.4$ & 0.31 & 1.4 & $0.67-2.94$ & 0.37 \\
\hline Histological type (Undifferentiated type) & 1.55 & $0.61-3.94$ & 0.35 & 2.95 & $1.05-8.29$ & $0.041^{*}$ \\
\hline $\mathrm{T}$ classification $(\mathrm{pT} 3 / 4)$ & 2.43 & $0.87-6.79$ & 0.09 & 0.82 & $0.22-2.93$ & 0.75 \\
\hline Venous invasion (present) & 3.06 & $1.64-5.71$ & $0.0004^{*}$ & 2.22 & $1.05-4.65$ & $0.036^{*}$ \\
\hline Lymphatic invasion (present) & 3.94 & $1.22-12.7$ & $0.02 *$ & 1.29 & $0.3-5.53$ & 0.74 \\
\hline Node involvement (present) & 1.53 & $1.03-2.28$ & $0.036^{*}$ & 1.22 & $0.63-2.37$ & 0.56 \\
\hline Distant metastasis (present) & 5.41 & $2.89-10.1$ & $<0.0001^{*}$ & 2.04 & $0.91-4.6$ & 0.08 \\
\hline High mGPS status ( $2 v s$. others) & 5.17 & $2.87-9.34$ & $<0.0001 *$ & 5.45 & $2.58-11.5$ & $<0.0001 *$ \\
\hline
\end{tabular}

\#The median age at surgery is 74 years in this cohort. HR: Hazard ratio. ${ }^{*} p<0.05$.

Table IV. Multivariate analysis for predictors of overall survival in Mie University cohort.

\begin{tabular}{|c|c|c|c|c|c|c|}
\hline \multirow[t]{2}{*}{ Variables } & \multicolumn{3}{|c|}{ Univariate } & \multicolumn{3}{|c|}{ Multivariate } \\
\hline & HR & $95 \% \mathrm{CI}$ & $p$-Value & HR & $95 \% \mathrm{CI}$ & $p$-Value \\
\hline Gender (Male) & 0.91 & $0.64-1.3$ & 0.62 & 0.78 & $0.54-1.12$ & 0.17 \\
\hline Age $\geq 67$ (median) $)^{\#}$ & 1.19 & $0.84-1.69$ & 0.32 & 1.29 & $0.91-1.84$ & 0.15 \\
\hline Tumor location (Rectum) & 1.06 & $0.74-1.5$ & 0.76 & 0.99 & $0.69-1.42$ & 0.94 \\
\hline Histological type (Undifferentiated type) & 0.73 & $0.47-1.12$ & 0.15 & 0.71 & $0.45-1.11$ & 0.13 \\
\hline $\mathrm{T}$ classification $(\mathrm{pT} 3 / 4)$ & 5.36 & $2.95-9.72$ & $<0.0001 *$ & 2.49 & $1.26-4.93$ & $0.009^{*}$ \\
\hline Venous invasion (present) & 2.92 & $1.93-4.43$ & $<0.0001 *$ & 1.39 & $0.84-2.3$ & 0.19 \\
\hline Lymphatic invasion (present) & 3.12 & $1.68-5.8$ & $0.0003^{*}$ & 0.61 & $0.28-1.35$ & 0.22 \\
\hline Node involvement (present) & 3.4 & $2.36-4.9$ & $<0.0001 *$ & 2.07 & $1.38-3.1$ & $0.0004^{*}$ \\
\hline Distant metastasis (present) & 8.12 & $5.68-11.6$ & $<0.0001^{*}$ & 6.2 & $4.19-9.16$ & $<0.0001 *$ \\
\hline High mGPS status ( $2 v s$. others) & 2.89 & $1.92-4.36$ & $<0.0001^{*}$ & 3.15 & $2.06-4.84$ & $<0.0001 *$ \\
\hline
\end{tabular}

${ }^{\#}$ The median age at surgery is 67 years in this cohort. HR: Hazard ratio. ${ }^{*} p<0.05$.

Furthermore, considering that most patients with advanced disease have already received postoperative chemotherapy, these high-risk CRC patients with high preoperative mGPS might need additional supportive treatment besides conventional systemic chemotherapy to further improve their prognosis.

In this regard, another major finding of the present study is the potential advantage of FO-enriched nutrition as an anti-SIR therapy during the postoperative course of CRC. Cancer cachexia, accompanied by wasting skeletal muscle and malnutrition, is a frequent problem in patients with various types of cancer especially during chemotherapeutic treatment and disease progression. Furthermore, several studies revealed that one of the major pathogenic factors in cancer cachexia is SIR via tumor-host interaction, and that this was intimately correlated with deterioration of functional status, quality of life, and poor prognosis in CRC $(36,37)$. Interestingly, our study demonstrated that a preoperative status of elevated CRP level and hypoalbuminemia was significantly correlated with dysregulation of each factor during the postoperative course, and that these factors are highly involved in tumor development. Based on these findings, a management protocol for SIR via tumor-host interaction during the postoperative course is urgently needed to improve the prognosis of CRC patients with a high preoperative mGPS score. Although nutritional intervention has been generally recognized as a recommended treatment option in patients with cancer cachexia (38, 39), an effective nutritional intervention for cancer cachexia has never been clarified. Eicosapentaenoic acid (EPA), a fish oil supplement, is one of the potential candidates for treatment of SIR-induced cancer cachexia in patients with malignant disease $(23,40,41)$. A previous study successfully demonstrated a significant increase in body weight in patients with gastrointestinal cancer or lung cancer who received daily supplementation of $2 \mathrm{~g}$ EPA (23). A recent study from another 
Table V. Multivariate analysis for predictors of overall survival in CRC patients with Stage III/IV disease from the Iga Ueno cohort.

\begin{tabular}{|c|c|c|c|c|c|c|}
\hline \multirow[t]{2}{*}{ Variables } & \multicolumn{3}{|c|}{ Univariate } & \multicolumn{3}{|c|}{ Multivariate } \\
\hline & HR & $95 \% \mathrm{CI}$ & $p$-Value & HR & $95 \% \mathrm{CI}$ & $p$-Value \\
\hline Gender (Male) & 1.22 & $0.61-2.43$ & 0.58 & 0.9 & $0.4-2.07$ & 0.81 \\
\hline Age $\geq 73(\text { median })^{\#}$ & 0.88 & $0.46-1.7$ & 0.7 & 0.66 & $0.3-1.46$ & 0.3 \\
\hline Tumor location (Rectum) & 1.08 & $0.57-2.05$ & 0.82 & 1.36 & $0.61-3.07$ & 0.45 \\
\hline Histological type (Undifferentiated type) & 2.2 & $0.85-5.71$ & 0.1 & 4.66 & $1.52-14.3$ & $0.007 *$ \\
\hline $\mathrm{T}$ classification $(\mathrm{pT} 3 / 4)$ & 3.22 & $0.44-23.6$ & 0.25 & 1.8 & $0.22-14.8$ & 0.59 \\
\hline Venous invasion (present) & 2.19 & $1.0-4.79$ & $0.049 *$ & 2.46 & $1.01-6.0$ & $0.048^{*}$ \\
\hline Lymphatic invasion (present) & - & - & 0.96 & - & - & 0.95 \\
\hline Node involvement (present) & 0.72 & $0.35-1.5$ & 0.39 & 0.59 & $0.21-1.63$ & 0.31 \\
\hline Distant metastasis (present) & 4.0 & $2.04-7.85$ & $0.0001 *$ & 2.1 & $0.9-4.89$ & 0.09 \\
\hline High mGPS status ( $2 v s$. others) & 4.68 & $2.4-9.13$ & $<0.0001^{*}$ & 5.69 & $2.4-13.5$ & $0.0001^{*}$ \\
\hline
\end{tabular}

\#The median age at surgery is 73 years in this cohort. HR: Hazard ratio. ${ }^{*} p<0.05$.

Table VI. Multivariate analysis for predictors of overall survival in CRC patients with Stage III/IV disease from the Mie University cohort.

\begin{tabular}{|c|c|c|c|c|c|c|}
\hline \multirow[t]{2}{*}{ Variables } & \multicolumn{3}{|c|}{ Univariate } & \multicolumn{3}{|c|}{ Multivariate } \\
\hline & HR & $95 \% \mathrm{CI}$ & $p$-Value & HR & $95 \% \mathrm{CI}$ & $p$-Value \\
\hline Gender (Male) & 0.95 & $0.64-1.42$ & 0.81 & 0.74 & $0.49-1.13$ & 0.16 \\
\hline Age $\geq 66$ (median) $)^{\#}$ & 0.89 & $0.6-1.33$ & 0.58 & 0.97 & $0.65-1.47$ & 0.89 \\
\hline Tumor location (Rectum) & 1.11 & $0.74-1.66$ & 0.61 & 0.83 & $0.55-1.27$ & 0.4 \\
\hline Histological type (Undifferentiated type) & 0.51 & $0.32-0.81$ & $0.005^{*}$ & 0.48 & $0.3-0.79$ & $0.003 *$ \\
\hline $\mathrm{T}$ classification $(\mathrm{pT} 3 / 4)$ & 5.34 & $1.69-16.9$ & $0.004 *$ & 3.83 & $1.17-12.6$ & $0.027 *$ \\
\hline Venous invasion (present) & 1.95 & $1.17-3.26$ & $0.011 *$ & 1.19 & $0.68-2.11$ & 0.54 \\
\hline Lymphatic invasion (present) & 1.63 & $0.52-5.16$ & 0.4 & 0.72 & $0.21-2.54$ & 0.61 \\
\hline Node involvement (present) & 0.93 & $0.54-1.6$ & 0.79 & 2.54 & $1.38-4.69$ & $0.003^{*}$ \\
\hline Distant metastasis (present) & 5.3 & $3.36-8.34$ & $<0.0001^{*}$ & 7.37 & $4.43-12.2$ & $<0.0001^{*}$ \\
\hline High mGPS status ( $2 v s$. others) & 3.82 & $2.37-6.16$ & $<0.0001 *$ & 3.31 & $2.0-5.49$ & $<0.0001^{*}$ \\
\hline
\end{tabular}

\#The median age at surgery is 66 years in this cohort. HR: Hazard ratio. $* p<0.05$.

group also showed that EPA-enriched nutrition prevented the chemotherapy-related decline in skeletal muscle mass in nonsmall cell lung cancer (NSCLC) patients compared with patients who did not receive EPA-enriched nutrition during chemotherapy (41). Findings of our preliminary study showed that FO-enriched nutrition suppressed SIR during tumor progression and increased skeletal muscle mass and lean body mass during systemic chemotherapy. In addition, FO-enriched nutrition provides survival benefits, especially in patients with advanced CRC with SIR (mGPS scores 1 and 2). Although a limitation of this study was the retrospective analysis of a small cohort, our findings combined with previous evidence clearly suggest that FO-enriched nutrition might control SIR induced by host-tumor interaction and maintain nutritional status during tumor progression in CRC patients.

In conclusion, this study provided novel evidence for the clinical relevance and potential feasibility of mGPS as a prognostic biomarker in CRC. Assessment of our developed
mGPS could identify patients with advanced CRC who have a poor prognosis and might be used to select appropriate candidates for FO-enriched nutrition intervention.

\section{Conflicts of Interest}

The Authors have no conflicts of interest to disclose.

\section{Acknowledgements}

The Authors thank all of medical and co-medical staffs in Iga city general hospital for their mindful cooperation.

\section{References}

1 Siegel R, Naishadham D and Jemal A: Cancer statistics, 2012. CA Cancer J Clin 62(1): 10-29, 2012.

2 Figueredo A, Coombes ME and Mukherjee S: Adjuvant therapy for completely resected stage ii colon cancer. Cochrane Database Syst Rev 3: Cd005390, 2008. 
Table VII. Correlation between pre- and post-operative serum levels of CRP and Albumin in CRC patients.

\begin{tabular}{|c|c|c|c|c|}
\hline & $\begin{array}{l}\text { Three months after surgery } \\
(\text { Mean } \pm \text { SD) }\end{array}$ & $p$-Value & $\begin{array}{c}\text { Six months after surgery } \\
(\text { Mean } \pm \text { SD })\end{array}$ & $p$-Value \\
\hline & \multicolumn{3}{|c|}{ Post-operative CRP levels (ng/ml) } & \\
\hline \multicolumn{5}{|c|}{ Pre-operative CRP levels } \\
\hline High $(\geq 0.5$ ng/ml) & $2.6 \pm 3.9$ & $0.002 *$ & $2.4 \pm 4.9$ & $0.0003^{*}$ \\
\hline \multirow[t]{2}{*}{ Low $(<0.5 \mathrm{ng} / \mathrm{ml})$} & $0.5 \pm 1.0$ & & $0.3 \pm 0.9$ & \\
\hline & \multicolumn{3}{|c|}{ Post-operative Albumin levels (g/dl) } & \\
\hline \multicolumn{5}{|c|}{ Pre-operative Albumin levels } \\
\hline $\operatorname{High}(\geq 3.5 \mathrm{~g} / \mathrm{dl})$ & $4.1 \pm 0.5$ & $0.0004 *$ & $4.1 \pm 0.5$ & $0.002 *$ \\
\hline Low $(<3.5 \mathrm{~g} / \mathrm{dl})$ & $3.6 \pm 0.7$ & & $3.6 \pm 1.0$ & \\
\hline
\end{tabular}

*One-way ANOVA, ${ }^{\#}$ Chi-square test, $p<0.05$.

Table VIII. Correlation between preoperative CRP status and pre-/post-operative skeletal muscle mass in CRC patients.

\begin{tabular}{|c|c|c|c|c|}
\hline & $\begin{array}{c}\text { Preoperative } \\
(\text { Mean } \pm \text { SD) }\end{array}$ & $p$-Value & $\begin{array}{l}\text { Six months after surgery } \\
(\text { Mean } \pm \text { SD })\end{array}$ & $p$-Value \\
\hline & \multicolumn{3}{|c|}{ Skeletal muscle mass $\left(\mathrm{cm}^{2}\right)$} & \\
\hline \multicolumn{5}{|c|}{ Pre-operative CRP levels } \\
\hline High $(\geq 0.5 \mathrm{ng} / \mathrm{ml})$ & $95.5 \pm 37.4$ & $0.024 *$ & $98.3 \pm 31.9$ & 0.058 \\
\hline \multirow[t]{2}{*}{ Low $(<0.5 \mathrm{ng} / \mathrm{ml})$} & $108.2 \pm 25.5$ & & $109.5 \pm 25.8$ & \\
\hline & \multicolumn{3}{|c|}{ Skeletal muscle mass $\left(\mathrm{cm}^{2}\right)$} & \\
\hline \multicolumn{5}{|c|}{ Pre-operative Albumin levels } \\
\hline $\operatorname{High}(\geq 3.5 \mathrm{~g} / \mathrm{dl})$ & $107.2 \pm 29.8$ & 0.11 & $109.6 \pm 27.4$ & $0.033^{*}$ \\
\hline Low $(<3.5 \mathrm{~g} / \mathrm{dl})$ & $94.0 \pm 35.2$ & & $94.8 \pm 27.4$ & \\
\hline
\end{tabular}

*Mann-Whiteney $U$-test, $p<0.05$.

3 Andre $\mathrm{N}$ and Schmiegel W: Chemoradiotherapy for colorectal cancer. Gut 54(8): 1194-1202, 2005.

4 Hanahan D and Weinberg RA: The hallmarks of cancer. Cell 100(1): 57-70, 2000.

5 Hanahan D and Weinberg RA: Hallmarks of cancer: The next generation. Cell 144(5): 646-674, 2011.

6 Guthrie GJ, Charles KA, Roxburgh CS, Horgan PG, McMillan DC and Clarke SJ: The systemic inflammation-based neutrophillymphocyte ratio: Experience in patients with cancer. Crit Rev Oncol Hematol 88(1): 218-230, 2013.

7 Mantovani A, Allavena P, Sica A and Balkwill F: Cancer-related inflammation. Nature 454(7203): 436-444, 2008.

8 Pages F, Galon J, Dieu-Nosjean MC, Tartour E, Sautes-Fridman $\mathrm{C}$ and Fridman WH: Immune infiltration in human tumors: A prognostic factor that should not be ignored. Oncogene 29(8): 1093-1102, 2010.

9 McMillan DC: The systemic inflammation-based glasgow prognostic score: A decade of experience in patients with cancer. Cancer Treat Rev 39(5): 534-540, 2013.
10 Toiyama Y, Miki C, Inoue Y, Tanaka K, Mohri Y and Kusunoki M: Evaluation of an inflammation-based prognostic score for the identification of patients requiring postoperative adjuvant chemotherapy for stage ii colorectal cancer. Exp Ther Med 2(1): 95-101, 2011.

11 Inoue Y, Iwata T, Okugawa Y, Kawamoto A, Hiro J, Toiyama Y, Tanaka K, Uchida K, Mohri Y, Miki C and Kusunoki M: Prognostic significance of a systemic inflammatory response in patients undergoing multimodality therapy for advanced colorectal cancer. Oncology 84(2): 100-107, 2013.

12 Miki C, Tonouchi H, Wakuda R, Hatada T, Inoue Y, Minato E, Kobayashi M and Kusunoki M: Intra-tumoral interleukin-6 downregulation system and genetic mutations of tumor suppressor genes in colorectal carcinoma. Cancer 94(5): 1584-1592, 2002.

13 Okugawa Y, Miki C, Toiyama Y, Yasuda H, Yokoe T, Saigusa S, Hiro J, Tanaka K, Inoue Y and Kusunoki M: Loss of tumoral expression of soluble il-6 receptor is associated with disease progression in colorectal cancer. Br J Cancer 103(6): 787-795, 2010. 
14 Koike Y, Miki C, Okugawa Y, Yokoe T, Toiyama Y, Tanaka K, Inoue $\mathrm{Y}$ and Kusunoki M: Preoperative c-reactive protein as a prognostic and therapeutic marker for colorectal cancer. J Surg Oncol 98(7): 540-544, 2008.

15 Toiyama Y, Fujikawa H, Koike Y, Saigusa S, Inoue Y, Tanaka K, Mohri Y, Miki C and Kusunoki M: Evaluation of preoperative c-reactive protein aids in predicting poor survival in patients with curative colorectal cancer with poor lymph node assessment. Oncol Lett 5(6): 1881-1888, 2013.

16 Toiyama Y, Miki C, Inoue Y, Minobe S, Urano H and Kusunoki $\mathrm{M}$ : Loss of tissue expression of interleukin-10 promotes the disease progression of colorectal carcinoma. Surg Today 40(1): 46-53, 2010.

17 Shirai Y, Okugawa Y, Hishida A, Ogawa A, Okamoto K, Shintani M, Morimoto Y, Nishikawa R, Yokoe T, Tanaka K, Urata H, Toiyama Y, Inoue Y, Tanaka M, Mohri Y, Goel A, Kusunoki M, McMillan DC and Miki C: Fish oil-enriched nutrition combined with systemic chemotherapy for gastrointestinal cancer patients with cancer cachexia. Sci Rep 7(1): 4826, 2017.

18 Edge SB, Byrd S, Compton CC, Fritz AG, Greene FL and Trotti A (eds): Ajcc cancer staging manual. 7th ed, New York: Springer, 2010.

19 Fearon K, Strasser F, Anker SD, Bosaeus I, Bruera E, Fainsinger RL, Jatoi A, Loprinzi C, MacDonald N, Mantovani G, Davis M, Muscaritoli M, Ottery F, Radbruch L, Ravasco P, Walsh D, Wilcock A, Kaasa $S$ and Baracos VE: Definition and classification of cancer cachexia: An international consensus. Lancet Oncol 12(5): 489-495, 2011.

20 McMillan DC: An inflammation-based prognostic score and its role in the nutrition-based management of patients with cancer. Proc Nutr Soc 67(3): 257-262, 2008.

21 Jho DH, Cole SM, Lee EM and Espat NJ: Role of omega-3 fatty acid supplementation in inflammation and malignancy. Integr Cancer Ther 3(2): 98-111, 2004.

22 Calder PC: Immunomodulation by omega-3 fatty acids. Prostaglandins Leukot Essent Fatty Acids 77(5-6): 327-335, 2007.

23 Fearon KC, Barber MD, Moses AG, Ahmedzai SH, Taylor GS, Tisdale MJ and Murray GD: Double-blind, placebo-controlled, randomized study of eicosapentaenoic acid diester in patients with cancer cachexia. J Clin Oncol 24(21): 3401-3407, 2006.

24 Barret M, Antoun S, Dalban C, Malka D, Mansourbakht T, Zaanan A, Latko E and Taieb J: Sarcopenia is linked to treatment toxicity in patients with metastatic colorectal cancer. Nutr Cancer 66(4): 583-589, 2014.

25 Jung HW, Kim JW, Kim JY, Kim SW, Yang HK, Lee JW, Lee KW, Kim DW, Kang SB, Kim KI, Kim CH and Kim JH: Effect of muscle mass on toxicity and survival in patients with colon cancer undergoing adjuvant chemotherapy. Support Care Cancer 23(3): 687-694, 2015.

26 Shibutani M, Maeda K, Nagahara H, Ohtani H, Sakurai K, Yamazoe A, Kimura K, Toyokawa T, Amano R, Kubo N, Tanaka H, Muguruma K, Ohira M and Hirakawa K: Significance of markers of systemic inflammation for predicting survival and chemotherapeutic outcomes and monitoring tumor progression in patients with unresectable metastatic colorectal cancer. Anticancer Res 35(9): 5037-5046, 2015.

27 Wakuda R, Miki C and Kusunoki M: Autoreactivity against interleukin 6 as a risk factor in elderly patients with colorectal carcinoma. Arch Surg 136(11): 1274-1279, 2001.

28 Canna K, McMillan DC, McKee RF, McNicol AM, Horgan PG and McArdle CS: Evaluation of a cumulative prognostic score based on the systemic inflammatory response in patients undergoing potentially curative surgery for colorectal cancer. $\mathrm{Br}$ J Cancer 90(9): 1707-1709, 2004.

29 Nozoe T, Matsumata T, Kitamura M and Sugimachi K: Significance of preoperative elevation of serum c-reactive protein as an indicator for prognosis in colorectal cancer. Am J Surg 176(4): 335-338, 1998.

30 McMillan DC, Canna K and McArdle CS: Systemic inflammatory response predicts survival following curative resection of colorectal cancer. Br J Surg 90(2): 215-219, 2003.

31 Crozier JE, McKee RF, McArdle CS, Angerson WJ, Anderson JH, Horgan PG and McMillan DC: The presence of a systemic inflammatory response predicts poorer survival in patients receiving adjuvant 5 -fu chemotherapy following potentially curative resection for colorectal cancer. Br J Cancer 94(12): 1833-1836, 2006.

32 Longo WE, Virgo KS, Johnson FE, Wade TP, Vernava AM, Phelan MA, Henderson WG, Daley J and Khuri SF: Outcome after proctectomy for rectal cancer in department of veterans affairs hospitals: A report from the national surgical quality improvement program. Ann Surg 228(1): 64-70, 1998.

33 Heys SD, Walker LG, Deehan DJ and Eremin OE: Serum albumin: A prognostic indicator in patients with colorectal cancer. J R Coll Surg Edinb 43(3): 163-168, 1998.

34 Longo WE, Virgo KS, Johnson FE, Oprian CA, Vernava AM, Wade TP, Phelan MA, Henderson WG, Daley J and Khuri SF: Risk factors for morbidity and mortality after colectomy for colon cancer. Dis Colon Rectum 43(1): 83-91, 2000.

35 Forrest LM, McMillan DC, McArdle CS, Angerson WJ and Dunlop DJ: Evaluation of cumulative prognostic scores based on the systemic inflammatory response in patients with inoperable non-small-cell lung cancer. Br J Cancer 89(6): 1028-1030, 2003.

36 Thoresen L, Frykholm G, Lydersen S, Ulveland H, Baracos V, Prado CM, Birdsell $\mathrm{L}$ and Falkmer U: Nutritional status, cachexia and survival in patients with advanced colorectal carcinoma. Different assessment criteria for nutritional status provide unequal results. Clin Nutr 32(1): 65-72, 2013.

37 Prado CM, Lieffers JR, McCargar LJ, Reiman T, Sawyer MB, Martin L and Baracos VE: Prevalence and clinical implications of sarcopenic obesity in patients with solid tumours of the respiratory and gastrointestinal tracts: A population-based study. Lancet Oncol 9(7): 629-635, 2008.

38 von Haehling S and Anker SD: Prevalence, incidence and clinical impact of cachexia: Facts and numbers-update 2014. J Cachexia Sarcopenia Muscle 5(4): 261-263, 2014.

39 Bauer JD AS, Davidson WL, Hill JM, Brown T, Isenring EA: Evidence based practice guidelines for the nutritional management of cancer cachexia. Nutr Diet 63: S3-S32, 2006.

40 Pappalardo G, Almeida A and Ravasco P: Eicosapentaenoic acid in cancer improves body composition and modulates metabolism. Nutrition 31(4): 549-555, 2015.

41 Murphy RA, Mourtzakis M, Chu QS, Baracos VE, Reiman T and Mazurak VC: Nutritional intervention with fish oil provides a benefit over standard of care for weight and skeletal muscle mass in patients with nonsmall cell lung cancer receiving chemotherapy. Cancer 117(8): 1775-1782, 2011.

Received December 18, 2017

Revised January 17, 2018

Accepted January 19, 2018 